POS $\quad$ PROCEEDINGS

\title{
Constraining Lorentz invariance violations using the Crab pulsar TeV emission
}

\author{
Markus Gaug* \\ Unitat de Física de les Radiacions, Departament de Física, and CERES-IEEC, Universitat \\ Autònoma de Barcelona, E-08193 Bellaterra, Spain \\ E-mail: markus.gaug@uab.cat

\section{Daniel Garrido} \\ Unitat de Física de les Radiacions, Departament de Física, and CERES-IEEC, Universitat \\ Autònoma de Barcelona, E-08193 Bellaterra, Spain \\ E-mail: daniel.garrido@uab.cat
}

\section{for the MAGIC Collaboration}

Fast variations of gamma-ray flux from Active Galactic Nuclei and Gamma-Ray Bursts can constrain Lorentz Invariance Violation (LIV) because of the delayed (or advanced) arrival of photons with higher energies: this approach has lead to the current world-best limits on the energy scale of Quantum Gravity. Here we report on complementary constraints on LIV by studying the gamma-ray emission up to $\mathrm{TeV}$ energies from the Galactic Crab pulsar, recently discovered by the MAGIC collaboration. A likelihood analysis of the pulsar events reconstructed for energies above $400 \mathrm{GeV}$ finds no significant variation of the arrival time with energy, and 95\% CL limits are obtained on the effective LIV energy scale taking into account systematic uncertainties. Only a factor of about two less constraining than the current world-best limit on a quadratic LIV scenario, pulsars are now well established as a third and independent class of astrophysical objects suitable to constrain the characteristic energy scale of LIV.

35th International Cosmic Ray Conference - ICRC2017

10-20 July, 2017

Bexco, Busan, Korea

\footnotetext{
* Speaker.
} 


\section{Introduction}

Quantum Gravity (QG) models [1] can include spontaneous violation of the Lorentz invariance (LIV) $[2,3,4,5,6,7,8]$, which lead, among others, to energy dependent dispersion relations of the photon in vaccuum. While such effects are expected to become important at energies of the order of the Planck scale $\left(E_{P l}=\sqrt{\hbar c^{5} / G} \approx 1.22 \cdot 10^{19} \mathrm{GeV}\right)$, they can already manifest themselves at much lower energies, as tiny deviations Lorentz invariance, which accumulate once the photons travels very large distances [9]. Introducing an effective quantum gravity scale $E_{Q G_{n}}$, which may be of the order of the Planck energy or lower, the group velocity of photons of energy $E \ll E_{Q G_{n}}$ can be expressed as an expansion in powers of $E$ (see e.g. [9]), where:

$$
u_{\gamma}(E)=\frac{\partial E}{\partial p} \approx c \cdot\left[1-\xi_{n} \frac{n+1}{2}\left(\frac{E}{E_{Q G_{n}}}\right)^{n}\right] .
$$

Here, $\xi_{n}=+1$ stands for a subluminal scenario, while $\xi_{n}=-1$ characterizes a superluminal scenario, and $\xi_{n}=0$ if the corresponding order is forbidden ${ }^{1}$. We consider, here, terms with $n>0$, which produce energy dependent velocities, typically considered in time-of-flight experiments, and then the linear case of $n=1$ and the quadratic case $n=2$. Odd terms of $n$ violate $C P T$ [11], that's why the $n=2$ case may dominate if $C P T$ is conserved.

So far, energy-dependent arrival time variations have been studied using flares from Active Galactic Nuclei (AGNs) [12, 13], and the very fast flux variations of Gamma-ray bursts (GRBs) $[14,15]$. In the linear case of $E_{Q G_{1}}$, the latter have achieved sensitivities well beyond the Planck scale [14] which has been effectively excluded, also by searches for birefrigence [16, 17]. For this reason, limits constraining the quadratic case are now of greater interest.

Constraints on $E_{Q G_{1}}$ have already been obtained from the Crab pulsar starting from 1969 [18], and constantly improved since then [19,20]. Although the Crab pulsar is found many orders of magnitude closer to us than AGNs and GRBs, some of them observed at high redshifts, its pulsations repeat and this can be used to improve, over many periods, sensitivity to LIV.

\section{MAGIC observation of TeV emission from the Crab Pulsar}

The Major Atmospheric Gamma-ray Imaging Cherenkov system (MAGIC) is located at the Roque de los Muchachos observatory $\left(28.8^{\circ} \mathrm{N}, 17.8^{\circ} \mathrm{W}, 2200 \mathrm{~m}\right.$ a.s.1.), in the Canary Island of La Palma, Spain. The MAGIC system consisted of a single $17 \mathrm{~m}$-dish telescope during its first 5 years of operation [21]. In 2009, a second telescope was added in order to create a stereo system [22]. A major upgrade was carried out between 2011 and 2012 [23, 24].

The Crab Nebula, together with its Pulsar, which cannot be spatially separated so far by Imaging Atmospheric Cherenkov Telescopes (IACTs), has been observed by MAGIC in every possible hardware configuration since its very beginning. Being the brightest steady Very High Energy (VHE) gamma-ray source in the sky, it is regularly observed for calibration purposes and performance monitoring, leading to more than a thousand hours of total observation time. These data have been down-selected to slightly more than $300 \mathrm{~h}$ of excellent quality, including single telescope

\footnotetext{
${ }^{1}$ Eq. 1.1 neglects terms breaking rotation invariance which if there, would however imply some breaking of boost invariance as well [10]
} 
("mono") and dual telescope ("stereo") configurations. Precision time stamping was required to attribute a precise pulsar phase to each registered event, using ephemeres provided by the Jodrell Bank Observatory [25].

With these data, MAGIC has detected emission from the Crab Pular up to $0.5 \mathrm{TeV}$ for the main pulse $P 1$, and up to $1.5 \mathrm{TeV}$ for the inter-pulse $P 2$ [26], showing $544 \pm 92$ excess events for $P 2$ above $400 \mathrm{GeV}$. The spectrum of both pulses is consistent with a power-law, however a significant difference was found between the reconstructed spectral indices of $P 1$ and $P 2$, the latter being harder [26]. This unique set of data is used to test LIV.

\section{Maximum likelihood method}

We construct a maximum likelihood method following the approach of [27], further elaborated in [13, 14], and slightly adapted for the quadratic LIV effect. Two new parameters are defined: $\lambda_{1} \equiv 10^{19} \mathrm{GeV} / E_{Q G_{1}}$ and $\lambda_{2} \equiv 10^{12} \mathrm{GeV} / E_{Q G_{2}}$. The LIV effect under test Eq. 1.1 produces then a mean phase delay of ${ }^{2}$ :

$$
\Delta \phi_{n}=c_{n} \cdot\left(\lambda_{n} \cdot\left(\frac{E}{\mathrm{GeV}}\right)\right)^{n}
$$

with :

$$
\begin{aligned}
c_{1} & =\xi_{1} \cdot \frac{d_{\text {Crab }}}{c \cdot P_{\text {Crab }}} \cdot 10^{-19}\left(\mathrm{GeV}^{-1}\right) \\
c_{2} & =\xi_{2} \cdot \frac{3}{2} \frac{d_{\text {Crab }}}{c \cdot P_{\text {Crab }}} \cdot 10^{-24}\left(\mathrm{GeV}^{-2}\right)
\end{aligned}
$$

We use now the profile likelihood ratio method [28] to define a test statistic $D_{n}$ :

$$
D_{n}\left(\lambda_{n} \mid \boldsymbol{X}\right)=-2 \ln \left(\frac{\mathscr{L}\left(\lambda_{n} ; \widehat{\widehat{v}}\left(\lambda_{n}\right) \mid \boldsymbol{X}\right)}{\mathscr{L}\left(\widehat{\lambda}_{n} ; \widehat{v} \mid \boldsymbol{X}\right)}\right),
$$

of the pulsar dataset $\boldsymbol{X}=\left\{E_{i}^{\prime}, \phi_{i}^{\prime}, k_{i}\right\}$ and a set of nuisance parameters $v$. Here, $E_{i}^{\prime}$ is the reconstructed energy of each event $i, \phi_{i}^{\prime}$ its reconstructed phase and $k_{i}$ the observation period. Singlehatted parameters $\left\{\hat{\lambda}_{n}, \widehat{v}\right\}$ maximize the likelihood, while double-hatted parameters $\widehat{\hat{v}}$ are those that maximize $\mathscr{L}$ under the assumption $\lambda_{n}$.

The likelihood $\mathscr{L}$ takes the form of an extended likelihood [30]:

$$
\begin{aligned}
\mathscr{L}\left(\lambda_{n} ; v \mid \boldsymbol{X}\right)= & \mathscr{L}\left(\lambda_{n} ; f, \alpha, \phi_{P 2}, \sigma_{P 2} \mid\left\{\left\{\left\{E_{i}^{\prime}, \phi_{i}^{\prime}\right\}_{i=0}^{N_{k}^{O N}}\right\}_{m=0}^{N_{k}^{O F F}}\right\}_{k=0}^{N_{s}}\right) \\
= & P(v) \cdot \prod_{k=0}^{N_{s}} \exp \left(-g_{k}\left(\lambda_{n} ; v\right)-b_{k} \cdot \frac{1+\tau}{\tau}\right) \cdot \prod_{m=0}^{N_{k}^{O F F}} b_{k} . \\
& \cdot \prod_{i=0}^{N_{k}^{O N}}\left(g_{k}\left(\lambda_{n} ; v\right)+b_{k} / \tau\right) \cdot \mathscr{P}_{k}\left(E_{i}^{\prime}, \phi_{i}^{\prime} \mid \lambda_{n} ; v\right) .
\end{aligned}
$$

\footnotetext{
${ }^{2}$ The definition of $\lambda_{2}$ differs slightly from $[13,14]$, and it is now directly proportional to $1 / E_{Q G_{2}}$ (the quantity of interest), instead of $1 / E_{Q G_{2}}^{2}$.
} 
Here, $N_{s}$ denotes the number of observation periods, $N_{k}^{O N}$ and $N_{k}^{O F F}$ the number of events in the $P 2$ $O N$ pulse region and the background control $O F F$ regions for observation period $k$, while $g_{k}$ and $b_{k}$ are their expectation values, respectively. We used $\phi^{\prime} \in[0.3558,0.4495]$ to define the $O N$ region (optimized through simulations), $\phi^{\prime} \in[0.52,0.87]$ for the $O F F$ region [31], and $E^{\prime} \in[0.4,7] \mathrm{TeV}$. This choice for the $O N$ region excludes contributions of $P 1$ and, practically, all possible contributions from bridge emission [32]. The ratio of phase width of the $O F F$, divided by the one of the $O N$ region is labelled $\tau$. The background expectation values $b_{k}$ are direct nuisance parameters, while the signal expectation contains the flux normalization $f$ as nuisance parameter. A possible probability density function (PDF) for the nuisance parameters, known from external measurements, is labelled $P(v)$. The set of nuisance parameters contains, apart from the $b_{k}$ : the $P 2$ flux normalization $f$, its spectral index $\alpha$, the mean pulse position $\phi_{P 2}$ and its width $\sigma_{P 2}{ }^{3}$.

The PDF of event $i$ is a combination of PDFs for signal (a pulsar event: $S_{k}\left(E_{i}^{\prime}, \phi_{i}^{\prime} \mid \lambda_{n} ; v\right)$ ), or the (interpolated) spectral energy distribution of the background, $h_{k}\left(E^{\prime}\right)$ (see e.g. [33]), for the $k$-th data subsample, respectively:

$$
\mathscr{P}_{k}\left(E_{i}^{\prime}, \phi_{i}^{\prime} \mid \lambda_{n} ; v\right)=\frac{b_{k} / \tau \cdot h_{k}\left(E^{\prime}\right)+g_{k}\left(\lambda_{n} ; v\right) \cdot S_{k}\left(E_{i}^{\prime}, \phi_{i}^{\prime} \mid \lambda_{n} ; v\right)}{g_{k}\left(\lambda_{n} ; v\right)+b_{k} / \tau} .
$$

The normalization constants of $S_{k}, g_{k}$, depends on all nuisance parameters and on $\lambda_{n}$. The signal PDF, $S_{k}\left(E_{i}^{\prime}, \phi_{i}^{\prime} \mid \lambda_{n} ; v\right)$, is written as:

$$
S_{k}\left(E_{i}^{\prime}, \phi_{i}^{\prime} \mid \lambda_{n} ; v\right)=\frac{\Delta t_{k} \int_{0}^{\infty} R_{k}\left(E \mid E_{i}^{\prime}\right) \cdot \Gamma_{P 2}(E, f, \alpha) \cdot F_{P 2}\left(\phi_{i}^{\prime}, E \mid \lambda_{n} ; \phi_{P 2}, \sigma_{P 2}\right) \mathrm{d} E}{g_{k}\left(\lambda_{n} ; v\right)}
$$

Here, $\Delta t_{k}$ denotes the effective observation time for each $k$-th data subsample; $R_{k}$ is the product of the effective collection area and the (inverted) energy re-distribution function to obtain a photon of true energy $E$, given its reconstructed energy $E^{\prime}$. Both obtained from Monte-Carlo simulations. The $P 2$ pulsar spectrum $\Gamma_{P 2}$ has been chosen as:

$$
\Gamma_{P 2}(E)=f \cdot\left(E / E_{\mathrm{dec}}\right)^{-\alpha} \cdot \exp \left(-E / E_{b}\right) \quad \mathrm{TeV}^{-1} \mathrm{~cm}^{-2} \mathrm{~s}^{-1}
$$

according to $[26]^{4}$. The pulsar phaseogram model $F_{P 2}$ is computed as:

$$
F_{P 2}\left(\phi_{i}^{\prime}, E \mid \lambda_{n} ; \phi_{P 2}, \sigma_{P 2}\right)=\frac{1}{\sqrt{2 \pi} \sigma_{P 2}} \cdot \exp \left[-\frac{\left(\phi_{i}^{\prime}-\phi_{P 2}-\Delta \phi\left(E \mid \lambda_{n}\right)\right)^{2}}{2 \sigma_{P 2}^{2}}\right],
$$

where the observed width $\sigma_{P 2}$ contains contributions from the intrinsic pulse width and the instrumental phase resolution, both considered Gaussian in nature ${ }^{5}$, while $\Delta \phi$ denotes the hypothetical phase delay produced by LIV (Eq. 3.1).

\footnotetext{
${ }^{3}$ Nuisance parameters might also include additional asymmetry parameters, a spectral cutoff, or other variables parameterizing a different pulse model.

${ }^{4}$ The analysis performed in [26] excludes a possible spectral cutoff below $700 \mathrm{GeV}$.

${ }^{5} \mathrm{An}$ intrinsic Lorentzian pulse shape has been investigated as well, yielding similar results.
} 


\section{Results}

The profile likelihood algorithm (Eq. 3.4) has been applied to the MAGIC Crab Pulsar data set [26], using the TMinuit class of ROOT [34] for the minimization. The minima of the profile likelihood were found close to zero in all cases (see Fig. 1). Table 1 displays the nuisance parameters obtained at the minimum, all compatible with those obtained in [26].

\begin{tabular}{ccc}
\hline $\begin{array}{c}\text { Nuisance } \\
\text { parameter }\end{array}$ & Result & Unit \\
\hline$\widehat{f}$ & $6.3 \pm 0.7$ & $\left(\cdot 10^{-10} \mathrm{TeV}^{-1} \mathrm{~cm}^{-2} \mathrm{~s}^{-1}\right)$ \\
$\widehat{\alpha}$ & $2.81 \pm 0.07$ & 1 \\
$\widehat{\phi}_{P 2}$ & $0.403 \pm 0.003$ & 1 \\
$\widehat{\sigma}_{P 2}$ & $0.015 \pm 0.003$ & 1 \\
\hline
\end{tabular}

Table 1: Nuisance parameter values at the minima of $\lambda_{1,2}$. Uncertainties are statistical only and obtained from the diagonal elements of the covariance matrix, as estimated by TMinuit.
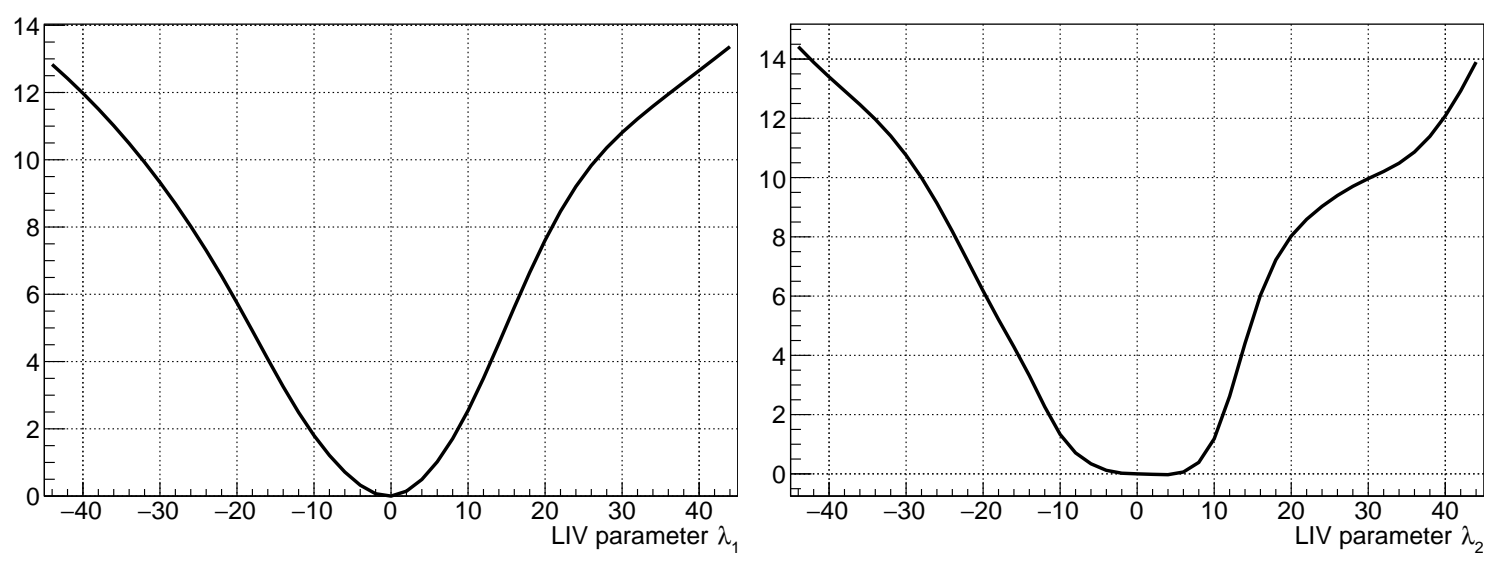

Figure 1: Left: test statistic (Eq. 3.4) as a function of $\lambda_{1}$, right: as a function of $\lambda_{2}$.

\section{Discussion and Conclusions}

Lower limits at $95 \%$ confidence level (CL) have been obtained by evaluating the likelihood at $\tilde{D}_{n}=\Delta \tilde{D}_{n}^{95 \%}$, where $\Delta \tilde{D}_{n}^{95 \%}$ has been obtained from simulations and found to be slightly higher than the canonical value of $\Delta \tilde{D}_{n}^{95 \%}=2.705$ [29]. The difference is due to the nuisance parameters, which have been varied in the simulations, using the covariance matrix obtained from the likelihood applied to experimental data.

We studied systematic effects due to the insufficient knowledge, i.e. modelling of the likelihood with respect to the background estimation, possible shifts in the assumed scale for energy and flux, different pulse shapes, including asymmetric behaviour, different values for the cutoff energy $E_{b}$, possible residual contributions from bridge emission, and the uncertainty of the pulsar distance. 
All values add up quadratically to about $42 \%$ for the linear case, and $36 \%$ for the quadratic one. Energy dependent source-intrinsic effects could also be present, and their detailed analysis is planned for the future.

Table 2 shows the obtained limits, with and without systematic uncertainties.

\begin{tabular}{ccc}
\hline case & $95 \%$ CL limit (w/o systematic) & $95 \%$ CL limit (incl. systematics) \\
\hline$\xi_{1}=+1$ & $E_{Q G_{1}}>7.8 \cdot 10^{17} \mathrm{GeV}$ & $E_{Q G_{1}}>5.5 \cdot 10^{17} \mathrm{GeV}$ \\
$\xi_{1}=-1$ & $E_{Q G_{1}}>6.4 \cdot 10^{17} \mathrm{GeV}$ & $E_{Q G_{1}}>4.5 \cdot 10^{17} \mathrm{GeV}$ \\
$\xi_{2}=+1$ & $E_{Q G_{2}}>8.0 \cdot 10^{10} \mathrm{GeV}$ & $E_{Q G_{2}}>5.9 \cdot 10^{10} \mathrm{GeV}$ \\
$\xi_{2}=-1$ & $E_{Q G_{2}}>7.2 \cdot 10^{10} \mathrm{GeV}$ & $E_{Q G_{2}}>5.3 \cdot 10^{10} \mathrm{GeV}$ \\
\hline
\end{tabular}

Table 2: Obtained limits applying the profile likelihood method.

These limits are found well below experimental results obtained from GRBs [14] for the linear case. The quadratic case yields constraints only about a factor two weaker when compared with the current best limits [14].

Pulsar data have the advantage that they can be continuously accumulated and in this way sensitivity to LIV keeps improving. MAGIC is currently operating at its best sensitivity [24], which gives the possibility to take regular data of the Crab Pulsar, particularly at higher zenith angles, where sensitivity for $\mathrm{TeV}$ energy gamma-rays is better. We expect that a data set of 2000 hours of stereo data, a number within reach for the MAGIC collaboration, will ensure an improvement of the quadratic limit by a factor of two, making it competitive with existing results, e.g. the current Fermi-LAT limit [14]. Moreover, our profile likelihood can be combined with that from other sources, like AGNs and other experiments. In such a way, significantly improved constraints on LIV are well within reach in the next years.

\section{Acknowledgments}

We would like to thank the IAC for the excellent working conditions at the ORM in La Palma. We acknowledge the financial support of the German BMBF, DFG and MPG, the Italian INFN and INAF, the Swiss National Fund SNF, the European ERDF, the Spanish MINECO, the Japanese JSPS and MEXT, the Croatian CSF, and the Polish MNiSzW.

\section{References}

[1] Rovelli C., Quantum Gravity (Cambridge University Press), 2004.

[2] Kostelecký V. A. \& Samuel S., Phys. Rev. D, 39 (1989) 683.

[3] Burgess C. P. et al., JHEP 3 (2002) 043.

[4] Gambini R. \& Pullin J., Phys. Rev. D 59 (1999), arXiv:gr-qc/9809038

[5] Douglas M. R. \& Nekrasov N. A., Rev. of Modern Phys. 73 (2001) 977.

[6] Magueijo J. \& Smolin L., Phys. Rev. Lett. 88 (2002) 190403.

[7] Hamed-Arkani N. et al., JHEP 5 (2004) 074. 
[8] Hořava P., Phys. Rev. D 79 (2009) 084008.

[9] Amelino-Camelia G. \& Smolin L., Phys. Rev. D 80 (2009) 084017.

[10] Mattingly D., Living Reviews in Relativity 8 (2005) 5.

[11] Colladay D. \& Kostelecký V. A., Phys. Rev. D 58 (1998) 116002.

[12] Albert J. et al., Phys. Lett. B 668 (2008) 253.

[13] Abramowski A. et al., Astrop. Phys. 34 (2011) 738.

[14] Vasileiou V. et al., Phys. Rev. D 87 (2013) 122001,

[15] Amelino-Camelia G. et al., Nature 393 (1998) 763.

[16] Kislat F. \& Krawczynski H., Phys. Rev. D 95 (2017) 083013.

[17] Götz D. et al., MNRAS 444 (2014) 2776.

[18] Warner B. \& Nather R. E., Nature 222 (1969) 157.

[19] Kaaret P., A\&A 345 (1999) L32.

[20] Otte N., in 32 nd ICRC, Beijing, China (2011) Vol. 7. https: / / arxiv. org/abs/1208.2033

[21] Cortina J., Goebel F. \& Schweizer T. for the MAGIC Collaboration, in $31^{\text {st }}$ ICRC, Lodz, Poland (2009). http://arxiv.org/abs/0907.1211

[22] Aleksić J., et al., Astrop. Phys. 35 (2012) 435.

[23] Aleksić J. et al., Astrop. Phys. 72 (2016) 61.

[24] Aleksić J. et al., Astrop. Phys. 72 (2016) 76.

[25] Lyne A. \& Roberts M., Jodrell Bank Crab Pulsar Monthly Ephemeris (2014) http: //www. jb. man. ac.uk/ pulsar/crab.html.

[26] Ansoldi S. et al., A\&A 585 (2016) A133, arXiv:1510.07048

[27] Martinez M. \& Errando M., Astrop. Phys. 31 (2009) 226.

[28] Murphy S. A., \& van der Vaart A. W., J. Am. Stat. Ass. 95 (2000) 449.

[29] Olive K. \& Particle Data Group, Chin. Phys. C 38 (2014) 090001.

[30] Barlow R., NIM A 297 (1990) 496.

[31] Fierro J. M. et al., ApJ 494 (1998) 734.

[32] Aleksić J. et al., A\&A 565 (2014) L12.

[33] Aleksić J. et al., JCAP 2 (2014) 008.

[34] Brun R. \& James F., TMinuit Class Reference, https://root.cern.ch/root/html534/TMinuit.html 